\title{
Simplified blast simulation procedure for hazard mitigation planning
}

\author{
T. Tadepalli \& C. L. Mullen \\ Department of Civil Engineering, University of Mississippi, USA
}

\begin{abstract}
A simplified procedure for blast simulation is presented for the purpose of performing building vulnerability assessment in a framework consistent with natural hazards mitigation planning studies. A computational tool, FESIM, has been developed to perform simplified building damage simulation using a GUI driven set of algorithms. FESIM permits estimation of the dynamic response of SDOF and MDOF systems to arbitrary forcing functions, and includes a beam-column damage element for computation of nonlinear response of portal frames including laminated composite beams with varying material and laminate layout sequences. The procedure begins with the formation of stiffness and mass matrices for computation of natural modes and frequencies of the portal frames. Blast loading-time histories are then generated and SDOF elasto-plastic response is computed to determine P-I curves. Geospatial analysis is used to display blast pressure contours for scenario events affecting a complex of buildings. The damage to buildings and debris created are estimated using the P-I curves developed for the class of buildings in the complex, and losses are computed based on the occupancy, structural value and content value. The procedure is demonstrated for buildings located at the University of Mississippi being studied as part of a federally sponsored Disaster Resistant University project.

Keywords: FE Simulation, blast loading, GIS, mitigation planning.
\end{abstract}

\section{Introduction}

The design of military structures for blast loads follows well laid design guidelines. The same is not the case when it comes to designing civilian structures for blast resistance. The technology transfer from military to civilian spheres of design has yet not been fully realized. Most of the design and analysis tools are not accessible to civil planners and designers, whose primary focus is 
on urban structures. It is not feasible to conduct complex computational fluid dynamics (CFD) and finite element (FE) simulations for an urban environment consisting of a very large number of structures. The aim of this work is to provide urban planners, designers and engineers with a simple tool for risk assessment and blast hazard mitigation. The major steps in conventional natural hazards loss estimation methodology are followed in terms of hazard analysis, structural analysis (engineering demand parameters), damage analysis and loss analysis; however, only deterministic scenarios are assessed in this procedure (Fig. 1).

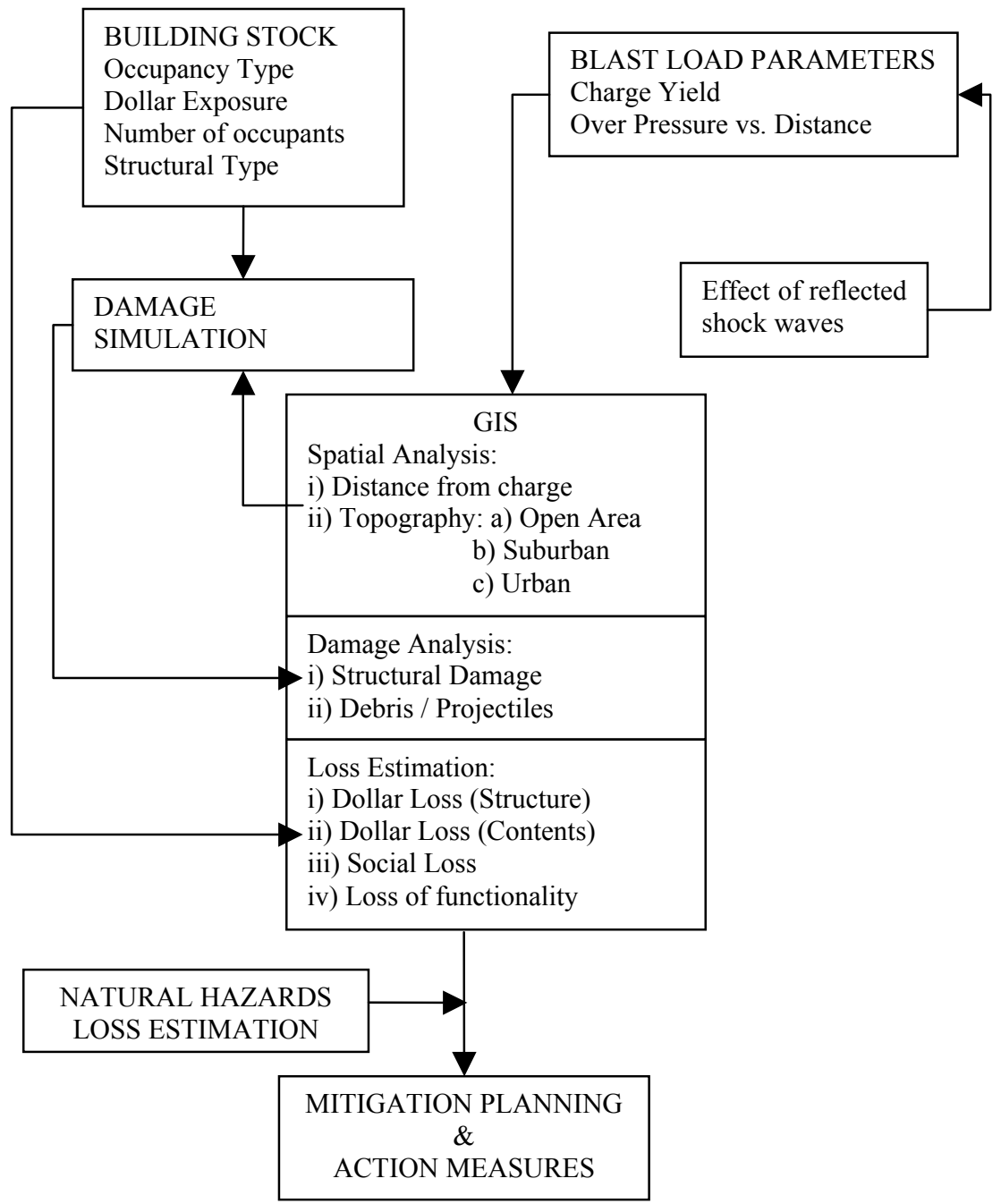

Figure 1: Proposed blast vulnerability assessment. 


\section{Hazard analysis}

The program provides blast overpressure versus distance curves, scaled for various charge yields of TNT based on empirical network type equations [1]. An equivalent triangular load is computed for subjecting the SDOF system to blast loading. For a given charge yield and stand off distance, the peak over pressure and impulse are computed from the scaling law.

The peak overpressures are modified based on the terrain surrounding the building. The contribution of the reflected shocks is determined based on how close the neighbouring structures are to the building(s) of interest. As of now, this is assigned to the structure by defining the terrain as open, suburban (buildings within $100 \mathrm{~m}$ of each other) or urban (buildings within $30 \mathrm{~m}$ of each other). Later versions of this program will perform spatial analysis to compute distances and orientations between the structures and compute the contributions of the reflected shock waves more accurately.

The amount of debris and fragments generated are computed based on the percentage of glazing on the exposed surfaces, from empirical formulae [1].

\section{Structural analysis}

An interactive computational simulation tool called FESIM which permits an interface between modal vibration analysis testing and finite element (FE) damage prediction has been developed, for composite structures dynamic response analysis. The tool operates in the Matlab (The Mathworks, Inc., Natick, MA) computing environment and is useful for advanced undergraduate/graduate instruction and has been developed in a computational framework that enables a variety of research activities. The FESIM framework includes a variety of time domain based linear and nonlinear dynamic analyses, which can initiate from either experimental modal analysis or FE formulation for structural systems made of advanced composite materials.

The instructional FESIM tool currently provides a 2D frame element for simulating linear static and dynamic analysis of frame structural systems. The research oriented tool is capable of simulating nonlinear dynamic behavior of 3D frame, solid, and shell systems. A graphical user interface (GUI) (Fig. 2) has been created to enable simple and direct input by the user including structural property matrices for small instructional problems.

\subsection{Section tangent stiffness integration and time varying flexural damage simulation in FESIM}

For the use of FESIM in an FE environment, a section damage model based on Euler-Bernoulli beam theory has been implemented [2, 3]. Sections are discretized into non-overlapping area patches (constant width rectangles for 2D case) for purposes of integration of stiffness and mass matrices.

A mixed interpolation strategy using linear shape functions for axial response and cubic (Hermitian) shape functions for flexural response is adopted to avoid shear locking that occurs with isoparametric shape functions. 


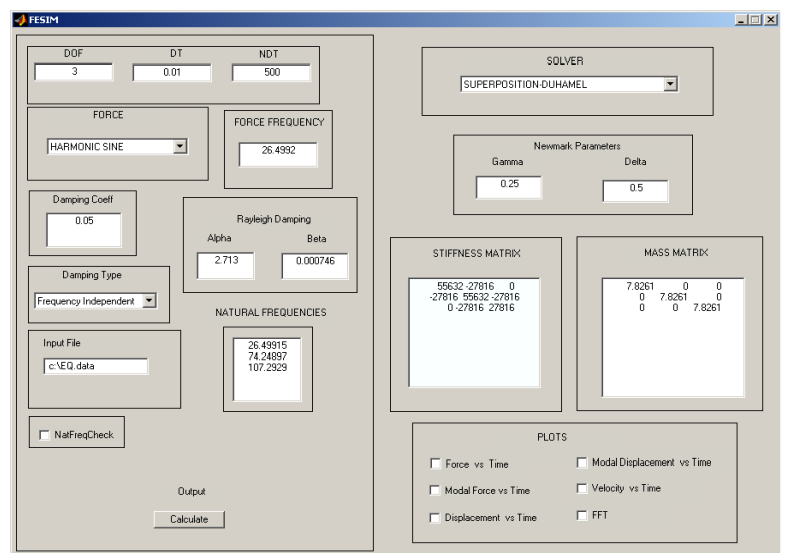

Figure 2: $\quad$ FESIM GUI shows interactive input and output.

The section stiffness and mass are computed at two Gauss integration points along the length of the element in order to minimize computational effort and storage. The shifting of neutral axis due to damage is also neglected.

The time varying strain versus nodal displacement vector for cross sections located at position $x$ on the neutral axis at time $\mathrm{t}$ is obtained from the strain compatibility on the section. The tangent stiffness matrix of the element is obtained by:

$$
{ }^{t} \mathbf{K}=\int_{V^{e}} \mathbf{B}^{T} \cdot{ }^{t} D^{N L} \cdot \mathbf{B} \cdot d V
$$

where

$$
\begin{aligned}
\boldsymbol{B}(x) & =\left[N_{1, x}^{L} ; y \cdot N_{1, x x}^{H} ; \mathrm{y} \cdot N_{3, x x}^{H} ; N_{2, x}^{L} ; y \cdot N_{2, x x}^{H} ; \mathrm{y} \cdot N_{4, x x}^{H}\right] \\
{ }^{t} D^{N L} & ={ }^{t} E=\frac{\partial\left[{ }^{t} \sigma_{1}\right]}{\partial\left[{ }^{t} \varepsilon_{1}\right]}
\end{aligned}
$$

The integration in (1) is first performed over the section and then over the length using numerical integration:

$$
{ }^{t} \mathbf{K}=\int_{L}{ }^{t} \mathbf{K}^{A} \cdot d x_{1}=\sum_{i=1}^{2}{ }^{t} \mathbf{K}^{A i}\left(x_{i}\right) \cdot w_{i}
$$

where ${ }^{t} \mathbf{K}^{A i}=\int_{A i} \mathbf{B}^{T}\left(x_{i}\right) \cdot{ }^{t} E \cdot \mathbf{B}\left(x_{i}\right) \cdot d A$

For the general case where the variation of the tangent modulus ${ }^{t} E$ with time and location is not defined by an explicit relation, (2) must be integrated numerically. For the $i^{\text {th }}$ integration point (section), a typical element of the tangent section stiffness matrix is: 


$$
{ }^{t}\left[\mathbf{K}_{22}\right]^{A i}=\left[N_{1, x x}^{H} \cdot N_{1, x x}^{H} \sum_{j=1}^{n a}{ }^{t} E_{j} \cdot I_{j}\right]_{i}
$$

where ${ }^{t} E_{i j}=$ tangent modulus at centroid of area patch $\mathrm{j}$ in section $\mathrm{i}$ at time $\mathrm{t}$ $I_{j}=I_{j 0}+y^{2} \cdot A_{i j}=2$ nd moment of area patch, $A_{i j}$, about $\mathrm{z}$-axis

\subsection{Application to incremental plastic collapse analysis of a cantilever beam}

A methodology for nonlinear incremental analysis [4] has been implemented in FESIM using the 2D frame element [3]. At each load increment, the local strain at each area patch centroid is computed, and the corresponding stress is interpolated from the material stress-strain data. This allows for definition of nonlinear material properties for each individual area patch. The tangent modulus is used to compute the stiffness matrix as described above. The modified Newton-Raphson iteration is used to compute the global equilibrium state.

FESIM simulation of the response of a cantilever beam loaded to collapse is performed using a multi-linear, elastoplastic material constitutive law, and the values obtained compare favorably with analysis using ABAQUS (HKS, Inc., Providence, RI) when sufficient numbers of area patches are used.

The section approach used in the stiffness integration allows the damage element to output data at all area patches, which is useful in visualizing the progression of damage both through the thickness and along the length. To illustrate this, a model of the cantilever is used to show the penetration of the elastic-plastic boundary [5] until formation of a plastic hinge at the fixed end (Fig. 3). The model only required two elements to accomplish the result shown. The routine is also useful in computing the yield displacements of frame structures through a pushover analysis.

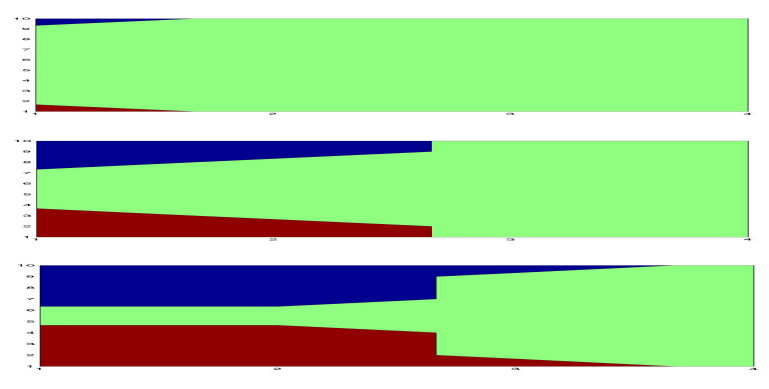

Figure 3: FESIM output for plastic hinge progression through the length and depth of a cantilever beam. 


\subsection{SDOF elastoplastic time history analysis}

The nonlinear dynamic response of elastoplastic systems is computed using the Newmark Method. The response of a SDOF system to a triangular impulse with varying rise times is provided as an example (Fig. 4). The peak response is observed to increase with decreasing rise time (tr) for a constant impulse.

Detailed nonlinear dynamic FE modelling of the Student Union building on the Oxford campus of the University of Mississippi was carried out as part of a research project examining seismic vulnerability of campus buildings (Fig. 5) [6]. The FE model is used here to develop the lumped parameters for an equivalent SDOF system. At present a SDOF oscillator having yielding bilinear force-displacement response characteristics is utilized for computing pressureimpulse (P-I) curves used in simulating blast response (Fig.4).

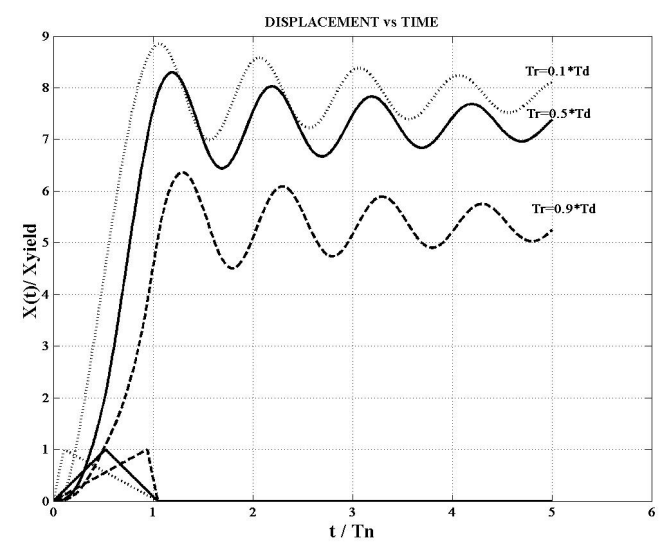

Figure 4: FESIM output for elasto-plastic SDOF system with varying rise time.

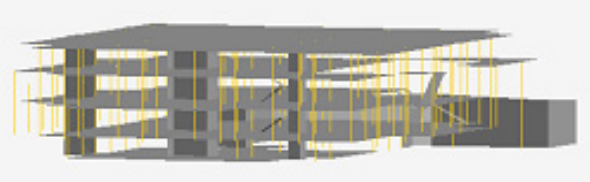

Figure 5: Detailed nonlinear FEA model of the Student Union Building.

\section{Pressure-Impulse (P-I) Curves}

P-I curves are also known as isodamage curves. Each curve represents a certain value for the ductility ratio. For various values of time duration of the impulse 
(td), the time of rise (tr), peak load and the impulse are varied. The maximum values of each response are computed, and if they match a certain desired value of the ductility ratio, they are plotted against the normalized peak load and normalized impulse $[7,8]$.

When a structural type is assigned to a building, pre-computed P-I curves corresponding to that structural type are assigned to the building. Thus, the P-I curves play a role analogous to fragility curves in seismic vulnerability assessment. The P-I curves (Fig. 6) for the Student Union building have been computed for ductility ratios of one, two and three.

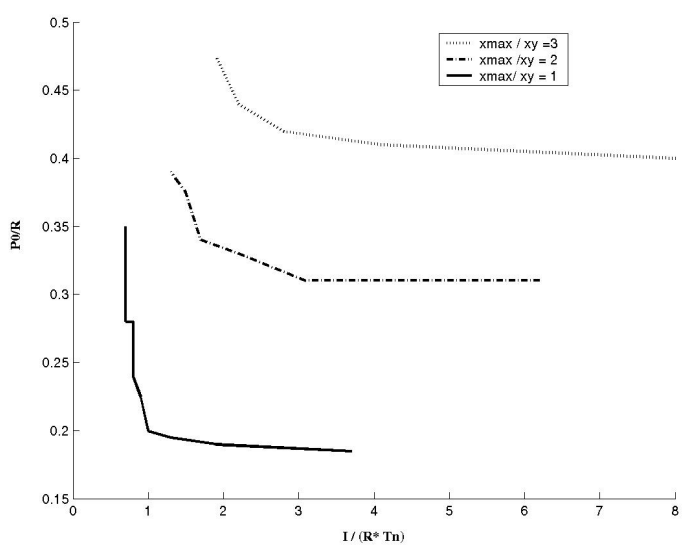

Figure 6: $\quad$ P-I curves for Student Union building.

\section{Geographical Information Systems (GIS)}

GIS provides the geospatial analysis for the proposed procedure. The GIS model for blast scenarios (Fig. 1) is similar to that used in HAZUS-MH for seismic, wind and flood scenarios. The advantage of geospatial analysis is that the user can see all the processes, relationships and linkages between various parts of the database. A building database may be populated with data obtained from field surveys. Building data relevant to direct physical damage estimation includes structural type, occupancy, number of storeys, glazing (glass, brick veneer) percentage of total exposed building surface area. For socio-economic impact, the dollar replacement value, contents value and the maximum number of occupants associated with each building are included in the building inventory.

A multi-hazard mitigation project [9] sponsored through a nation-wide Disaster Resistant University program is currently underway on the Oxford campus of the University of Mississippi. The proposed blast simulation procedure is applied to a group of the campus buildings to illustrate the potential benefits. A deterministic scenario is considered involving an explosive having a charge yield of $500 \mathrm{~kg}$ TNT that is detonated from a vehicle parked on campus. 
The results and implications are visualized on a GIS map that overlays photos and geospatial relationships of a small group of buildings surrounding the vehicle (Fig. 7)

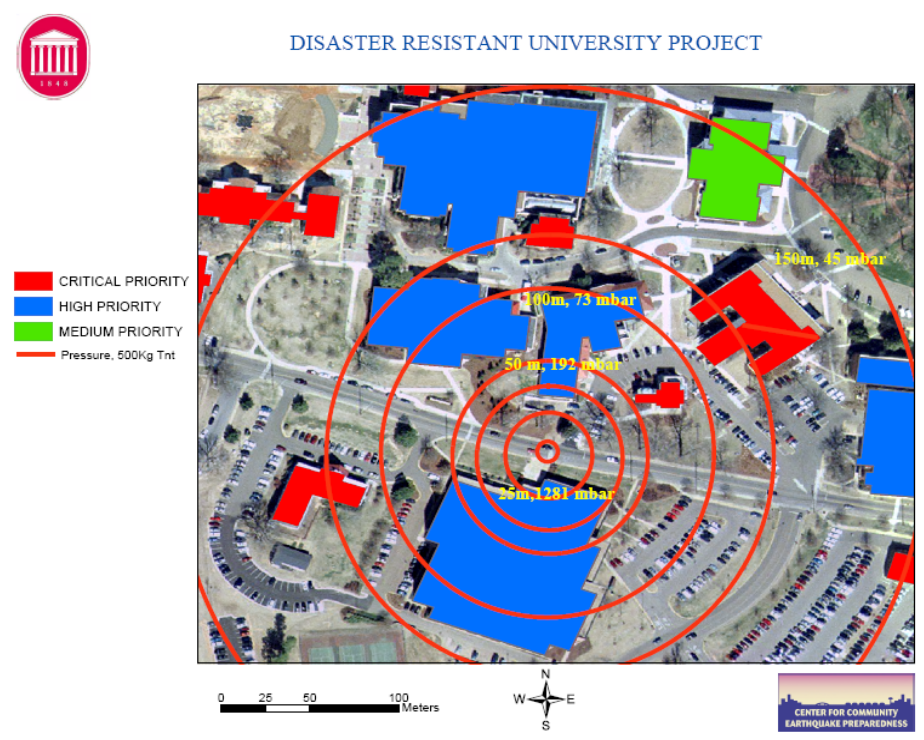

Figure 7: Hypothetical blast scenario on university campus.

\section{Damage analysis}

Once the correlation between a blast loading event and the damage is established, the response of equivalent SDOF systems is used to develop P-I curves for various structural types. The ratio of peak displacement to the yield displacement of the structure, also known as the maximum displacement ductility demand, is utilized as a parameter for determining the damage to a given building. For example, in the case of steel structures, a ratio of 3 is considered to correspond to moderate damage [8]. The damage levels are quantified as low, moderate and high. Once the degree of damage in a building due to a blast event is determined, the performance levels of the building are classified into immediate occupancy, life safety and collapse prevention.

\section{Loss estimation}

The loss due to a blast event is computed using a methodology similar to that used for seismic loss estimation. The premise is that the range of damage states which might occur due to a blast is similar to that which might occur during earthquakes. Once the damage due to a blast event is determined, the loss is estimated as a percentage of the building dollar value, the contents value, social 
loss and loss of functionality. The dollar loss is computed based on the current replacement value, even though the buildings may be constructed in stages. The contents value can be estimated from the building inventory. The average number of people occupying the building at a peak occupancy level is used to determine the social loss. The dollar loss due to non-functionality is computed based on the dollar revenue generated per day by the facility.

Once the risk of a specified level of blast occurring in various locations is estimated and critical facilities are identified based on their economic, administrative, emergency management, operational, and social importance, action measures may be identified that might mitigate the projected losses. Some measures call for more detailed analysis such as hardening of building structures. Other measures relating to policy making and non-intrusive actions such as event control and provision for barriers or buffer zones may be accomplished directly using the simplified procedure.

\section{References}

[1] Kinney, G.F., and Graham, K.J., Explosive Shocks in Air, $2^{\text {nd }}$ Ed., Springer Verlag, 1985.

[2] Mullen, C.L., and Cakmak, A.S., "A practical damage element for seismic analysis of RC structures," unpublished work in partial fulfilment of the degree of Doctor of Philosophy, Department of Civil Engineering and Operations Research, Princeton University, 1996.

[3] Tadepalli, T.P., "Interactive computational tools for simulating linear dynamic response and nonlinear quasi-static damage in composite structures," thesis submitted for the degree of Master of Science, Department of Civil Engineering, University of Mississippi, 2003.

[4] Bathe, K.J., Finite Element Procedures, Prentice-Hall, Inc., 1996.

[5] Lubliner, J., Plasticity Theory, Prentice-Hall, Inc., 1998.

[6] Swann, C.T., Mullen, C.L., et al, "Evaluation of earthquake effects on selected structures and utilities at the University of Mississippi: A mitigation model for universities," final report submitted to MEMA, 1999.

[7] Li, Q.M. and Meng, H., "Pressure-impulse diagram for blast loads based on dimensional analysis and single-degree-of-freedom model," Journal of Engineering Mechanics, 128 [1], 2002.

[8] "Design, materials, and connections for blast-loaded structures," ABS Consulting Ltd, Research Report 405, Health and Safety Executive, 2000.

[9] "Natural hazard mitigation plan of the University of Mississippi, Lafayette County, Mississippi," Center for Community Earthquake Preparedness, 2006 (www.olemiss.edu/orgs/ccep). 